\title{
Greenweb in Land Enhancement Policy of Enna Province. A DRSA Valuation Pattern in WebGIS Interaction Practice
}

\author{
Salvatore Giuffrida ${ }^{1, a}$, Filippo Gagliano ${ }^{2, b}$ and Maria Rosa Trovato ${ }^{3, c}$ \\ ${ }^{1}$ Via E. Albanese, 28 - 90139 - Palermo, Italy \\ ${ }^{2}$ Via Mons. Palermo, 7 - 94015 - Piazza Armerina (EN), Italy \\ ${ }^{3}$ Viale A. Doria, 6 - 95125 - Catania, Italy \\ asgiuffrida@dica.unict.it, bfmgagliano@gmail.com, mrtrovato@dica.unict.it
}

Keywords: Greenways, WebGIS, DSRA, Axiological Approach, Qualitative Assessment, Land assessment, Land planning.

\begin{abstract}
The Enna Province is characterized by a low degree of economic, infrastructural and industrial development. Its hilly territory is a fair combination of many different and integrated landscapes. These conditions suggest the possibility of a sustainable development pattern in which the slow mobility, because of the low level of land infrastructures, can become one of the most important network for the land-value communication.

The study applies an axiological approach, useful for the subsequent land planning practice, including a qualitative valuation model and an interactive multi-criteria tool combining Web-GIS and DRSA (Dominance Rough Set Approach) patterns.

The valuation model is based on an axiological square in which four kinds of appreciation are taken into account. A WBS pattern explains in detail each appreciation, so that every piece of the green-web can be characterized and assessed within a general framework oriented to provide the aggregate value of the path to which they relate.

The DRSA tool is used to generate the preferences structure of the target segments users. It is used as basis for extracting and the processing data. It allows to identify the preferences structure to support the WebGIS tools to generate the green way that best meet the user's preferences.
\end{abstract}

\section{The Enna Province}

The Enna Province occupies the centre of Sicily, and within its hilly territory converge some important land signs in Sicily, the ridges of three major mountain chains, Nebrodi, Madonie and Erei, that had been dividing Sicily into three administrative districts, the "Vali" during the Islamic and the Norman periods (827-1072): "Val Demone", "Val di Noto" and "Val di Mazzara" (Fig. 1).
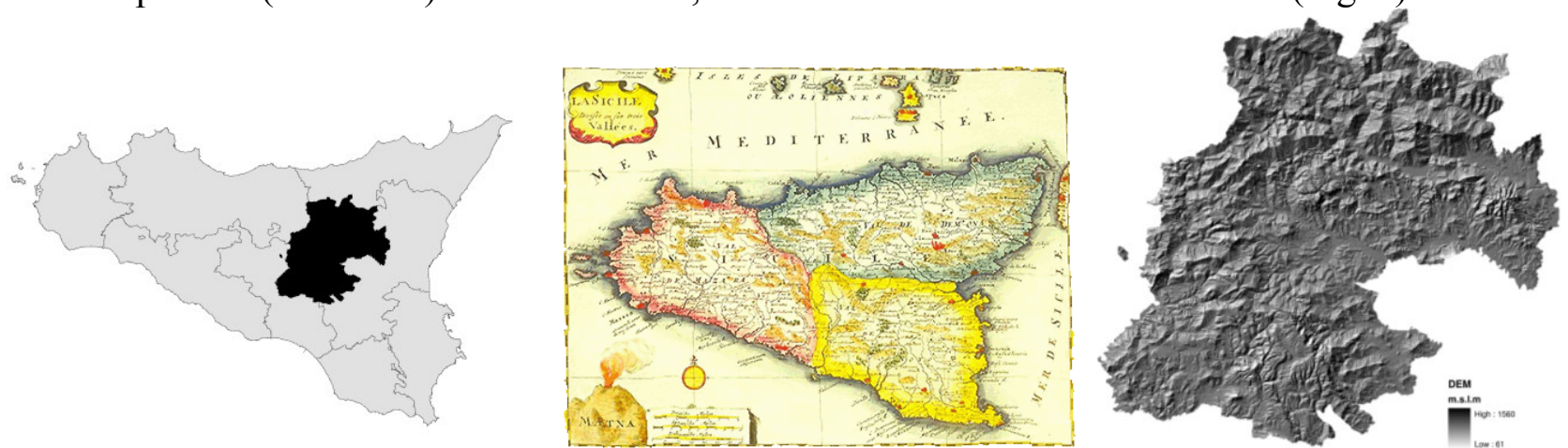

Fig. 1 - Geographic framing, ancient "Vali" (administrative districts) and orography of the province of Enna.

As a consequence, this territory synthesises the most important features of the Sicilian landscape comprising: 280 archaeological sites; an uncontaminated landscape that can be considered the synthesis of the regional environment because of the fair integration between cultural-historical and morphological features; an environmental-natural heritage including an important hydrogeology system composed of several lake basins, 11 Oriented Nature Reserves with their specific features of 
flora and fauna; a remarkable mining and mineralogical heritage with industrial archaeology features; a fair synthesis between the agricultural-forest framework and the consequent arable-arboreal landscape; a rich anthropic landscape including many urban centres with relevant remains of several historic civilizations whose cultural and religious traditions are somehow recognisable up to now.

Nevertheless, some criticalities must be highlighted, and in particular: land instability phenomena; not rational land uses; a general trend to abandon the territory and the ancient urban centres; landslides; degradation and collapse of the lake basins; deforestation and desertification; intrusive infrastructures. In addition, the reserves aren't appropriately accessible because of the lack of: suitable delimitations, trails, information signs, access points, visitor centres; a general land marketing system.

1. The Integrated Land Plans, as means to implement in local areas the Operative Regional Plans; the ILPs (PIT in Italian) boost the bottom-up local economic development by matching the institutional aims and the purposes of the social and economic organizations.

2. LEADER II, a European program aimed to boost the rural development, by involving the local communities in innovative and multi-sectorial initiatives.

3. The Territorial Landscape Province Plan is the main and the most extensive and detailed planning tool for the general knowledge and the government of the territory. It is based on an advanced Geographical Information System describing the three main landscape component: abiotic, biotic and anthropic.

Each component indicates the enhancement of the green paths network as the means of a sustainable development pattern based on the land culture and information. As a consequence, signification, information, and communication [10] can be assumed as a general landscape re-production paradigm in which the land (in)form(ation) assumes the role of primary "substance of value", the main origin and result of a sustainable development pattern.

\section{Greenways: a culture as a method for territory enhancement}

Landscape, as a whole human experience, is strictly linked to sustainability, because of its holistic dimension [12], thus confirming it as a natural and cultural unity [2], [10]. Greenways, as both a physical infrastructure and a cultural approach to landscape [5], combine natural and cultural factors, and rational and creative approaches. A greenways network can assume a large bundle of functions such as connecting different anthropic land districts, promoting a cultural and economic upgrade of rural land, developing sustainability awareness [1], renovating the scale of values and preferences, as the Italian Greenways Association, founded in 1998, declares in its program [15].

Creating networks can be considered one of the main attitude of social value point [5]: greenways can be considered the channels which the land information gets through, and due to the difficult to compare costs and positive externalities, an interactive assessment model, involving planners [11] and users, could be helpful. Greenways are assumed as the physical communicative network of land, through which the users spread land information: the more users get through the network, the more land social value increases.

\section{Values, valuations and valorization: a semiotic and axiological approach}

A semiotic approach [3] to valuation can be assumed as the basis of an axiological method for planning. Landscape is a perceptual/communicative land fabric whose value-frame a green-web could be. A green-web is a network of high-value experiences so that two questions arise.

1. What do we mean as "value of a path"? The valuation process gets through the formalization of: some explicit criteria; a set of utility functions in order to transform performances into specific valuations; a weight system; a procedure for aggregating all the elementary valuations into the main criteria. Each greenway part can be considered a "signifier unit" as well as in a semiotic signification process, in which each signifier (the set of characteristics of a path, not the path in itself) implies a reference (the physical frame and its both natural and artificial components) and a meaning (the 
importance of them for someone or a specific community), so that no intrinsic value can be considered relevant. The value is strongly influenced by the user's profile and moreover by the textual unit in which it takes part as well. Therefore, the same object or performance can assume different values according to the different user's profiles. On the contrary, the same satisfaction can be achieved in many different (green)ways. So, the value of a path is given as the set of the valuations, properly aggregated, from the point of view of the different criteria.

2. Do actually paths among which to choose, currently exist? Our concern is a preliminary collection of information, valuations and planning indications aimed at realizing an interactive decision tool referring to the path which can be assembled according to the axiological user's profile. As a consequence, the paths among which to chose have to be composed by using parcels of the existing dirt roads: the system provides the aggregation of parcels that maximizes the function of the landscape-experiential value.

This is what we mean as an axiological approach, a value-centered and value-oriented vision, so that land cannot be meant as object and function, but as a bundle of combined values. The basic hypothesis is: the land social value can be distinguished into potential and current value: the first one is based on the occurrences (object and performances) which can be observed and measured; the second one depends on the appreciation of these resources by the users and then on their psychological and cultural determination, that is the axiological profile their choices are due to.

Accordingly, a specific tool has been realized; in the latter the user can express his or her preferences and communicate the satisfaction degree of the experiences, if carried out, so that the evaluator can adjust the tool: the user inputs his or her preferences into a form on the web-gis interface, basing on his or her specific axiological profile. The system proposes a group of paths which the user can further reduce to select the best one, by inserting more specific information about his or her wishes and expectations. The input form and the related preference pattern are inserted into three different sections, each of them each of them referred to one of three different approaches: 1 . Object, 2. Performance, 3. Axiological.

\section{Valuation pattern}

1. The web-form to fill in order to select the path, proposes a list including all kinds of the objects which are present in that part of land. The user selects the objects or the places - archaeological sites, monuments, panoramas, natural features - he wants to come across; the system makes a query and composes all the paths containing the kinds of objects indicated in the form.

2. The section of performances includes some functional characteristics of the path, that can be a) measurable performances, as the maximum length, slope, traffic road crossings, or b) valuable performances, as smoothness, hardness, riskiness etc.; the valuable ones are calculated by using the space analysis web-gis functions; the pattern reduces the previous selection so that the user can refine the query to select the best path.

Object and performance approaches can (or should) be used together: they actualize the most concrete and specific approach and do not require the involvement of a coordinated value system.

3. The section of value includes the weighs of the four main appreciations that the user, according to his/her profile, inserts. According to an axiological approach, objects and performances are relevant only in order to achieve a purpose which is referred to a value. The value is not attributed to objects or performances but to the capability of the path to satisfy some general instances when they are crossed; thus objects and performances have no value in themselves; the user assigns to them a value once connected by the path whose configuration is defined by assembling a certain number of path units, so that the value function is optimized. The value of the path is the weighed average score calculated going up the WBS from the leaves (indicators), through arms (subcriteria and criteria) to the roots (values).

The root-criteria of the pattern are taken from the axiological square [6] a general diagram in which four kinds of appreciation are connected by three kinds of relationship, complementary, contrary and contradictory. Practical (functionality), critical (efficiency and convenience), utopic (mythic, existential), playful (differences, surprise) appreciations can be distinguished. Each of them 
corresponds to specific traveller's profiles shown in the square (fig. 2, left), as detailed by WBS (fig. 2 , right) in which the contents of the valuation pattern are connected and organized by a progressive disaggregation in different criteria and subcriteria (the sub-sub criteria, the indicators and the 145 indices are omitted).

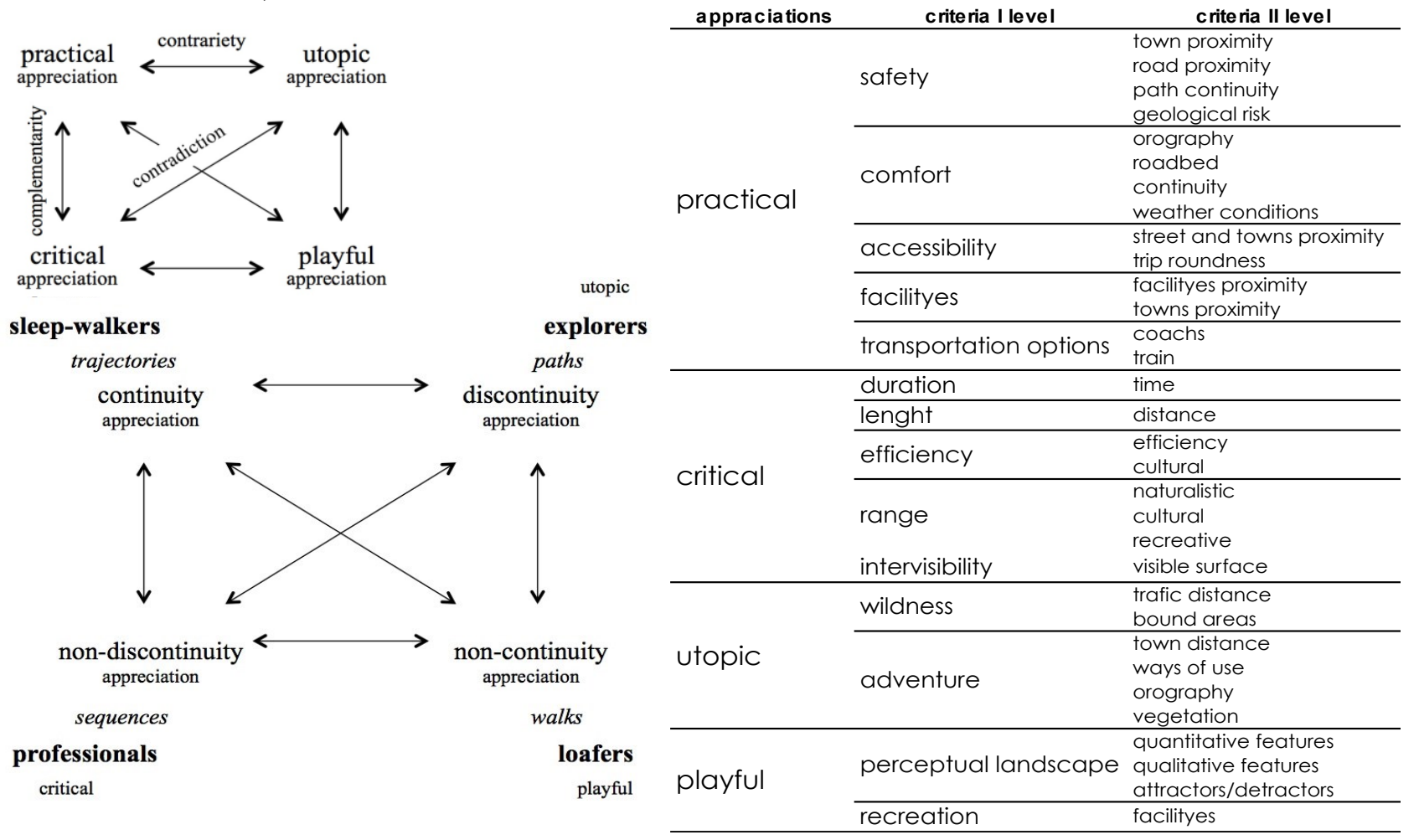

Fig. 2 - Traveller's profiles (Floch, 1995 modified) and the valuation pattern.

\section{Space analysis and GIS tools application}

A GreenWeb should be considered, from a topologic point of view, as a set of arcs and nodes linked into a reticular framework connecting the social land fabric. Each node is usually associated to a value function, but in this experience the value is traced to the path as a whole. Network Analyst extension is the tool which aggregates the path maximizing this value function.

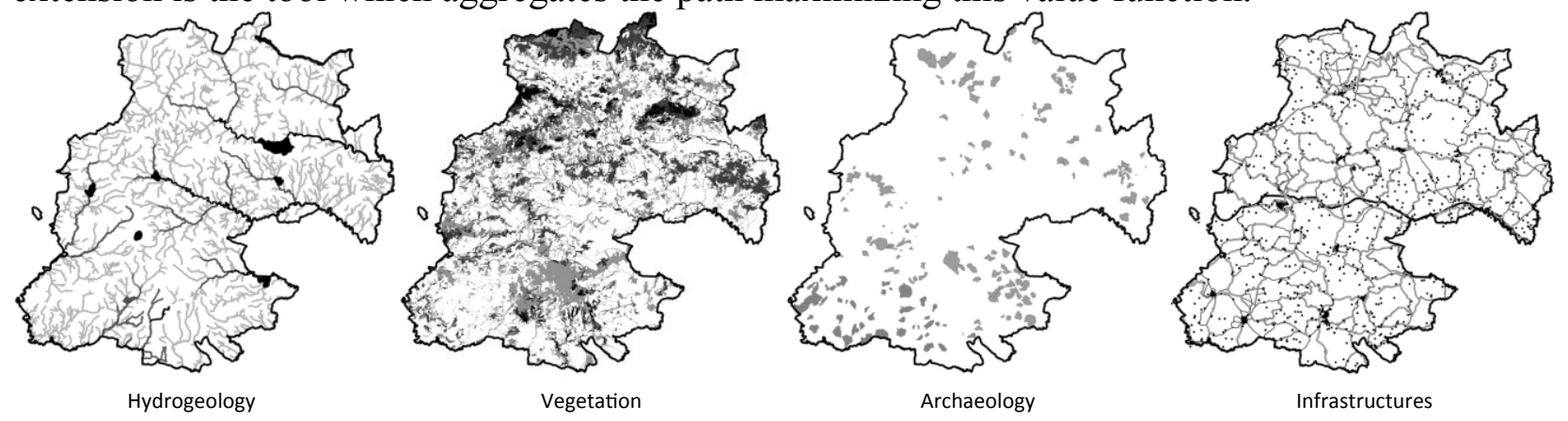

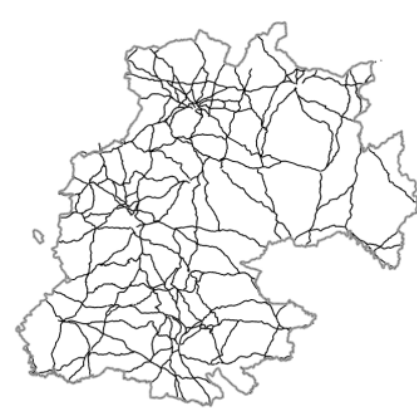

Royal trazzeras

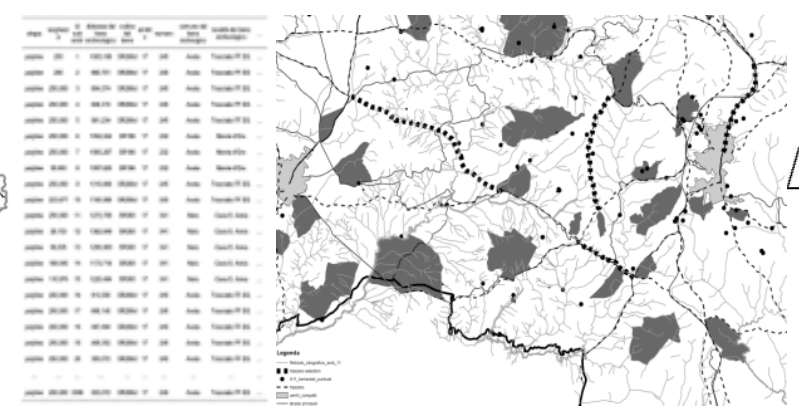

Database

GIS representation

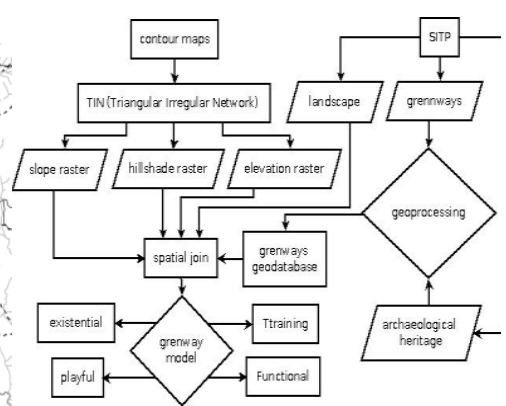

Logic scheme for path selection

Fig. 3 - Landscape values mapping and application of GIS tools 
The database includes the ancient road network as shown in IGM 1:50.000 maps in 1965; some groups have been distinguished: main (consular) roads, herds' roads, lanes; 2. old railways [13]; all of them have been geo-referenced. By means of the Spatial Join extension and the geoprocessing functions a new viability database has been implemented by dividing each road into $250 \mathrm{~m}$ long segments, so that a continuous greenway can be assembled by joining the arcs which maximize the value function. Spatial join and Range query are the two geometric operations more frequently used in the geographic data management. The spatial join is a relational join in which geometric attributes and space relations are used and imposed instead of alphanumeric ones. There are: the topologic join that is more speed if the storage structure is based on a set of layers; other join based on direction and distance (fig. 3).

\section{Interactive value adjustment pattern based on the DRSA approach}

The greenway can be considered a product-service for the users. To improve a recreational product-service it is necessary to identify an appropriate marketing strategy. The marketing management must coordinate the recreational demand with the local supply, in relation to the target segments of the users. The development of some new information and communication technologies (ICTs) help to coordinate the supply to demand, which is ever changing and more globalized. The web GIS is a ICTs tool that, if properly structured, is able to support the development of a Web 2.0 type, and therefore the tourism 2.0. The tools to support the development of a web GIS which is able to meet these requirements are: a data mining and an artificial intelligence tool that produces an output of the informational type for the product or service requested by the user. To support the extraction and the processing data, the study proposes the DRSA -Dominace Rough Set Approach and fuzzy sets (Greco, Masahiro and Slowinski, 2006). The DRSA tool is used to generate the preferences structure of the target segments users. The DRSA enables to generate a minimal set of decision rules in a neutral way [7]. By means of this minimal set $[8,9]$ it is possible to generate a preferences structure or perceptual-value structure for the user [14], [15] (Tab. 1). The identified preferences structure may to support the GIS tool and the Web GIS tool in generating the best solution for the user's "green way". The information data for the data mining are obtained from some feedback questionnaires which are present in the institutional web that uses the proposed web GIS tool. In particular, the questionnaires are proposed to users at the feedback button on the GIS Web's site. The construction of the database and data mining to support the choice of the green way constitutes a examples and a test for the model that we are studying. Surely, when the sample turns out to be more representative, the model will be able to generate a stable preferences structure by means of which implementing the data mining and generating, using an automatic action, an individual more satisfying path (Tabb. 2-4).

Tab. 1 - Decision rules

\section{Decision Rules}

1. If the level of importance for the efficiency of the route is medium and the level of importance for the recreational facilities is high then choose the playful profile;

2. If the level of importance for the efficiency of the route is medium and the level of importance for the recreational facilities is high then choose the playful profile;

3. If the level of importance for the distances is medium, the level of importance for the perceptual landscape is medium and the level of importance for the recreational facilities is high choose the playful profile;

4. If the level of the importance for the recreational facilities is high then choose the existential profile;

5. If the level of importance for the recreational facilities is medium then choose the critical profile;

6. If the level of the importance for the density of the events is medium then choose the critical profile;

7. If the level of importance for the adventure is medium then choose the critical profile

$$
\text { Tab. } 2 \text { - The preferences structure to support the critical profile }
$$

\section{The critical profile}

1. If the level of importance for the recreational facilities is medium then chooses the critical profile;

2. If the level of the importance for the density of the events is medium then chooses the critical profile;

3. If the level of importance for the adventure is medium then chooses the critical profile. 
Tab. 3 - The preference structure to support the existential profile

\section{The existential profile}

1. If the level of importance for the recreational facilities is high then chooses the existential profile.

Tab. 4 - The preferences structure to support the playful profile

\section{The playful profile}

If the level of importance for the efficiency of the route is medium and the level of importance for the recreational facilities is high then chooses the playful profile;

If the level of importance for the efficiency of the route is medium and the level of importance for the recreational facilities is high then chooses the playful profile;

If the level of importance for the distances is medium, the level of importance for the perceptual landscape is medium and for the recreational facilities is high then chooses the playful profile.

\section{Conclusions}

The multiple selection into the different areas of the web form allows to apply the different approaches, the object one (orange), the performances one (light green) and the axiological one (dark green) separately or simultaneously (fig. 4). Further inputs reduce the selected path or modify them. The DRSA extension helps to refine the appraisal pattern basing on the influence of each appreciation on the query.

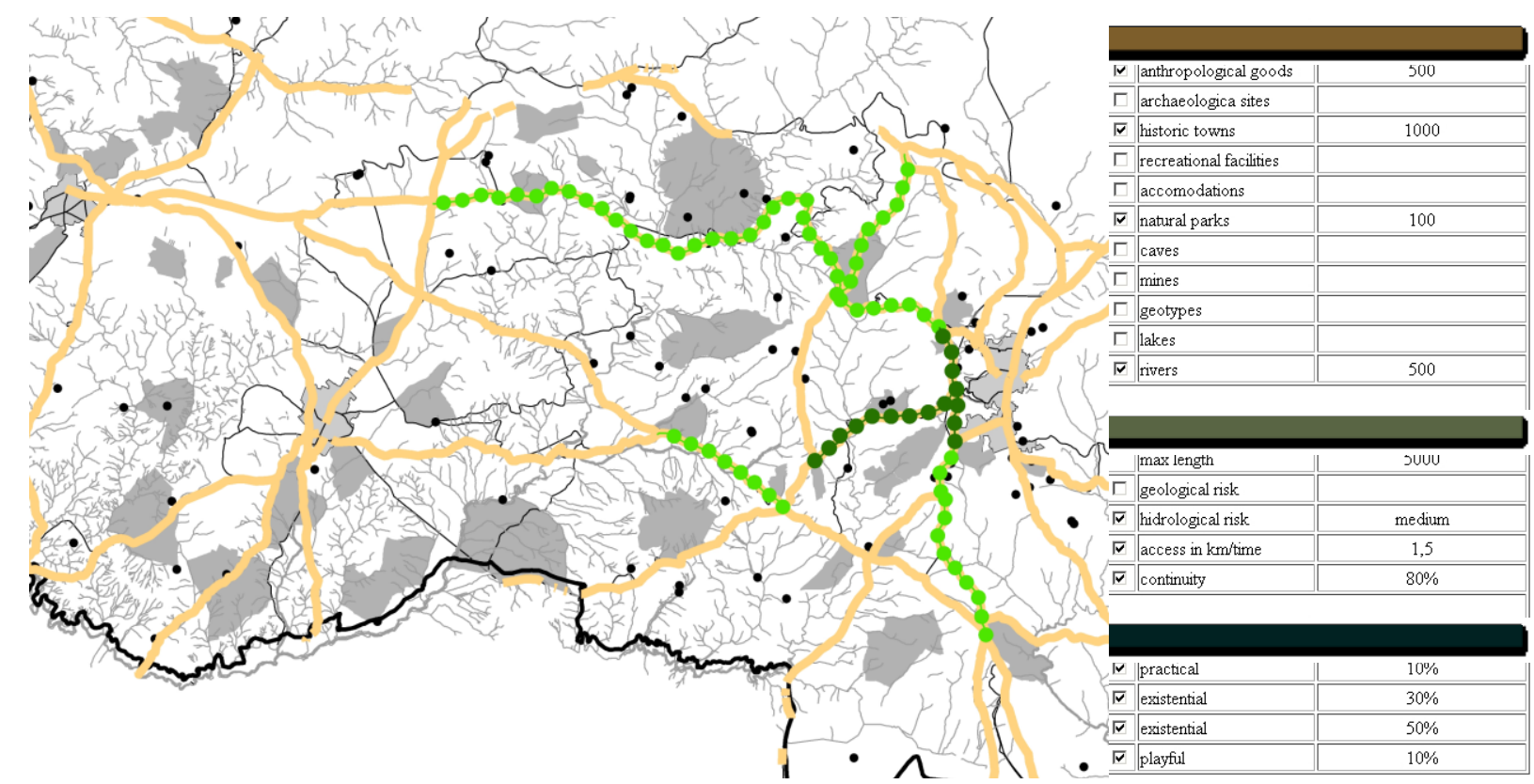

Fig. 4 - Results of the path section procedure carried out by applyign the three approaches.

A green-web is an immaterial infrastructure, a phase of the information cycle - information as "the form that informs" - whose origin is the organization of the land knowledge and the access to it through of a personalized consultation system.

Therefore the green-web can be considered composed of the knowledge system, the physical land support and the land values as produced by administration, planners, professionals and at last by the users through a recursive process of signification, information and communication (Rizzo, 1999). These three parts, between which the "value/valuation" is the most relevant one, are involved in the feedback process at the three levels of data/information, value/valuation, planning/communication.

At the first level the experience we have carried out has been a test about the connection between data and values, so that the knowledge system has been completely redrawn; values need some specific data, and an appropriate way of turning them into information. At the second level, the valuation one, the value system has been assumed as the matrix of the knowledge whose wide articulation has to be reduced to some axiological relationships, in order to create a shareable 
communication system: an axiological approach connects the data and plan levels. At the third level, the experimentation of the DRSA method has shown how it is possible to connect a valuation model to a planning approach; the interaction between user and decision-maker through the valuation model provides useful insights about what part of the land has to be enhanced and what supply chains need to be boosted for the general equalization purpose.

\section{References}

[1] J. Ahern: Greenways as planning strategy, Landscape and Urban Planning, No. 33 (1995)

[2] P. Dal Sasso, M. A. Ottolino: Greenway in Italy: Examples of Projects and Implementation, Journal of Agricultural Engineering, 1 (2011), pp. 29-39

[3] U. Eco: A Theory of Semiotics, Bloomington: Indiana University Press (1976)

[4] P. Dasgupta: Social Capital and Economic Performance: Analytic, in E. Ostrom and T.K. Ahn, eds., Critical Writings inEconomic Institutions: Foundations of Social Capital, Cheltenham, UK: Edward Elgar (2003)

[5] J. G. Fabos, J. Ahern (Eds.): Greenways: The beginning of an international movement. Elsevier, Amsterdam: 1-13 (1995)

[6] J. M. Floch: Semiotica, marketing e comunicazione, Milano, FrancoAngeli (1995)

[7] S. Greco, B. Matarazzo, R. Slowinski: Rough sets theory for multi criteria decision analysis, European Journal of Operational Research, No. 129. Elsevier Science, (2001), pp. 1-47

[8] S. Greco, B. Matarazzo, R. Slowinski: Dominance-Based Rough Set Approach to Knowledge Discovery (I) - General Perspective, in Zhong, N., Liu, J. (by), Intelligent Technologies for Information Analysis, Springer Verlag, Berlin, (2004a), pp. 513-552

[9] S. Greco, B. Matarazzo, R. Slowinski: Dominance-Based Rough Set Approach to Knowledge Discovery (II) - Extensions and Applications, in N. Zhong, J. Liu, (by), Intelligent Technologies for Information Analysis, Springer-Verlag, Berlin, (2004b), pp. 553-612

[10] F. Rizzo: Valore e valutazioni. La scienza dell'economia o l'economia della scienza. Milano, FrancoAngeli (1999)

[11] R. L. Ryan, J. G. Fabos, J. J. Allan: Understanding opportunities and challenges for collaborative greenway planning in New England, Landscape and Urban Planning, 76, (2006), pp. 172-191

[12] J. Stephenson: The Cultural Values Model: An integrated approach to values in landscapes, Landscape and Urban Planning, 84, (2008), pp. 127-139

[13] Superintendence of Cultural Heritage of the Province of Enna, AUD University of Catania Landscape Territorial Plan of the Province of Enna (2008)

[14] Sturiale, L., Trovato, M. R.: La percezione sociale a supporto della valutazione degli interventi di valorizzazione di una risorsa ambientale, Paysage/Topscape, No 9, (2010), pp.365-416

[15] A. Toccolini, N. Fumagalli, G. Senes: Greenways planning in Italy: the Lambro River Valley Greenways System, Landscape and Urban Planning, 76, (2006), pp. 98-111

[16] M. R. Trovato: A fuzzy measure of the ability of a real estate capital to increase in value. The real estate decision problem for Ortigia, In: Proceedings of XLI Meetings Ce.S.E.T (Rome), Appraisals. Evolving proceedings in global change, Firenze University Press., (2013), pp. 697-720 